\title{
Desenvolvimento de uma técnica de detecção de irregularidades ionosféricas usando dados GPS e Densidade in situ do Satélite C/NOFS.
}

\author{
*Silva, E. N.; Cueva, R. Y. C. \\ UEMA. \\ Copyright 2016, SBGf - Sociedade Brasileira de Geofísica \\ Este texto foi preparado para a apresentação no VII Simpósio Brasileiro de Geofísica, \\ Ouro Preto, 25 a 27 de outubro de 2016. Seu conteúdo foi revisado pelo Comitê Técnico \\ do VII SimBGf, mas não necessariamente representa a opinião da SBGf ou de seus \\ associados. É proibida a reprodução total ou parcial deste material para propósitos \\ comerciais sem prévia autorização da SBGf.
}

Programa de Pós-Graduação em Engenharia de Computação e Sistema - PECS, Universidade Estadual do Maranhão -

\section{Abstract}

It is proposed in this paper the study of the equatorial ionosphere and low latitude equatorial latitudes over the Brazilian region, using statistics and observational techniques of the spread $F$ with multi-instruments for the development of a technique to detect the trigger mechanism of ionospheric irregularities when present= For the development of this work we have the dataset like TEC data from GPS receivers, in-situ density from C/NOFS satelite. We look the understanding in order to be able to identify the plasma bubble electrodynamics and their generation.

\section{Introdução}

A ionosfera tem sido estudada durante vários anos por muitas técnicas de observação, irregularidades nela são detectadas pelas ionossondas e digissondas, radar de espalhamento coerente e incorente, cintilações nas transmissões GPS, luminescência atmosférica, foguetes (in situ) e satélites (in situ).

Os fenômenos de irregularidades de densidade eletrônica do plasma equatorial, observado pelos mais diversos tipos de instrumentos de sondagem ionosférica é denominado espalhamento F equatorial ("equatorial spread F-ESF'), ocorre principalmente durante a noite, provocando cintilações nos sinais de rádio emitidos por satélites, devido a variação da densidade de elétrons. Cada instrumento ou técnica é sensível a uma determinada escala de tamanho ou faixa de escalas, como mostra a Figura 1. Muitas das características do ESF podem ser explicadas pela teoria da instabilidade Rayleigh-Taylor (RT). ESF consiste em uma reviravolta gravitacional turbulenta da ionosfera em que densidades de plasma e domínios elétricos desenvolvem estruturas irregulares, com comprimentos de escala de centenas de quilômetros até dezenas de centímetros (Valladares et al., 2004). As ocorrências de ESF continuam sendo um problema desafiador para as pesquisas científicas e de predições no âmbito do clima espacial, assim como no sistema de navegação.

O aparecimento ou não do ESF pode resultar do aumento da deriva vertical do plasma, perto do pôr do sol. Tal processo chamado pico pré-reversão (PRE), que se dá pela rápida diminuição da condutividade elétrica da região $E$, produzindo campos elétricos de polarização. Assim que - PRE se desenvolve completamente, a anomalia de Appleton é intensificada, formando cristas onde a densidade de plasma é muito elevada e o efeito de cintilação é mais evidente quando comparado com o equador magnético e em altas latitudes. O PRE é um dos fatores mais importantes controladores do ESF, mas não é o único.

Valladares et al., (2004) mostraram que existe uma forte relação entre o nível de cintilação GPS com a diminuição do TEC (Conteúdo Eletrônico Total). Weber et al., (1996) 
Desenvolvimento de uma técnica de detecção de irregularidades ionosféricas.

também demonstraram relação entre a depleção do TEC e diminuição airglow $630.0 \mathrm{~nm}$, investigando as irregularidades chamadas bolhas ionosféricas.

A pesquisa aqui apresentada, consiste no desenvolvimento de uma técnica para detecção de irregularidades ionosféricas para a região brasileira nas proximidades de São Luís.

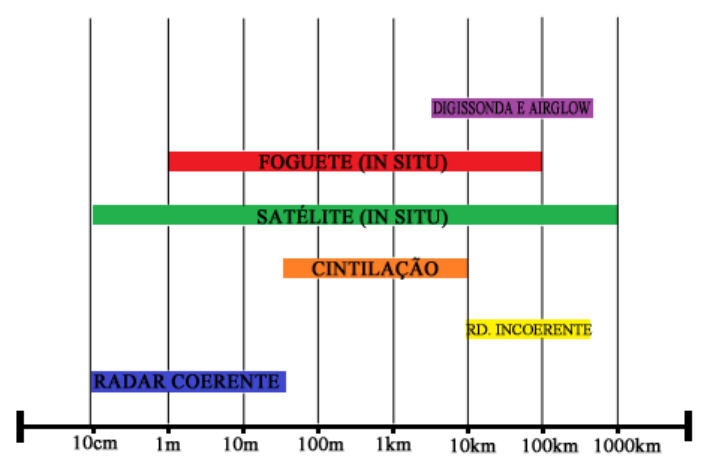

Figura 1. Relação entre a escala de comprimento das irregularidades da região $F$ e o tipo de técnica usada em sua detecção.

\section{Métodos/Problemas Investigados}

Estudar-se-á a propagação de bolhas de plasma sobre a região brasileira com o uso de dados TEC de receptores GPS e densidade in-situ do satélite C/NOFS quando as passagens são sobre as regiões de interesse. Em presença das bolhas de plasma na região o índice de cintilação S4 cresce e o valor do TEC apresenta depleção. Valladares et al., (2004) inferiram que a ocorrência de cintilações GPS, na verdade, correspondem a bolhas de plasma, ou depleções do TEC, que consistem em uma redução brusca do TEC seguido por uma recuperação para um nível próximo do valor TEC anterior à exaustão. No entanto, processos não-bolhas podem produzir variações rápidas no TEC, levando a uma identificação equivocada de depleções TEC, processos tais como a decadência noturna da camada $F$ e a redistribuição da densidade causado pelo efeito fonte também podem diminuir o valor do TEC, entretanto, estes tipos de processos tem ausência do segmento de recuperação.
A Figura 2 mostra o percurso do satélite C/NOFS (linha tracejada em azul) onde destaca-se o nível de cintilação (veja barra lateral direita) observado a uma altura aproximada de 400 km de altitude no dia 159 de 2011. Para esse dia o satélite encontrou registro de bolhas apenas próximo às 0 UTC, e no intervalo de $135^{\circ}$ a $160^{\circ}$ longitude leste, a figura mostra também a concordância entre o nível de cintilação (linha preta) e a depleção no TEC (linha vermelha), observa-se que a densidade de plasma se esgota e depois retorna a um valor próximo ao que possuía anterior a sua diminuição. Isso acontece porque o satélite ao atravessar a estrutura (bolha) encontra uma região de baixa densidade eletrônica, onde sua densidade pode ser reduzida entre 1 a 4 ordens de grandeza no interior da depleção. O índice de cintilação do satélite está acima do nível de ruído. Em base ao explicitado e com maiores considerações físicas será desenvolvido uma técnica para detecção de bolhas de plasma na região brasileira usando dados TEC da rede de receptores presentes (LISN, RBMC, IGS, etc), além dos dados de densidade in situ do satélite C/NOFS. Procurase também evidencias de mecanismos geradores de bolhas de plasma visando prever sua ocorrência.

\section{Resultados}

O trabalho desenvolvido além da detecção das irregularidades do ESF na região $F$ equatorial e de baixas latitudes, poderá ajudar a diagnosticar as possíveis causas dessas irregularidades, dando grande contribuição ao desenvolvimento de estudos na ionosfera.

Será desenvolvido um Algoritmo Automático em linguagem Matlab para detecção de bolhas de plasma que servirá como suporte para estudo de estruturas que derivam na ionosfera de forma horizontal e vertical. O uso do Matlab na criação do Algoritmo é de grande interesse pela facilidade de manuseio e pela linguagem amigável apresentada pelo mesmo, fornecendo grande dinâmica, 
Silva, E. N.; Cueva, R. Y. C.
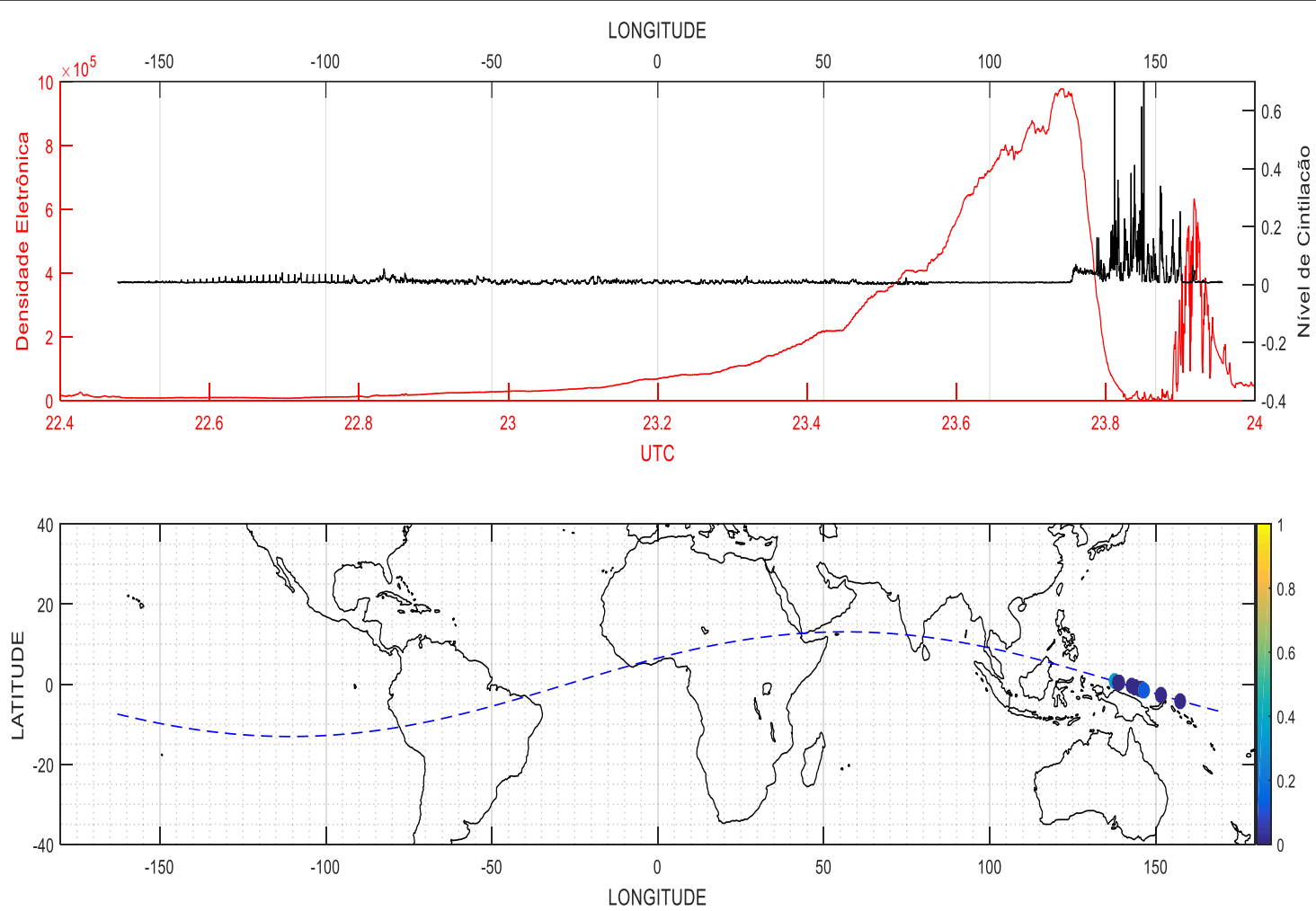

Figura 2. Esta figura mostra o percurso de um satélite C/NOFS (linha tracejada em azul)) onde destaca-se o nível de cintilação (veja barra lateral direita) observada a uma altura aproximada de $400 \mathrm{~km}$ de altitude no dia 159 de 2011, destacando a relação da depleção da densidade eletrônica com a cintilação de ondas eletromagnéticas emitidas pelo satélite, assim que ele atravessa as estruturas (bolhas).

poder de adaptação e melhoria do Algoritmo pelos diversos pesquisadores de ionosfera.

Os trabalhos a serem desenvolvidos irão acrescentar com o conhecimento da ionosfera equatorial e de baixas atitudes principalmente na América do Sul, trazendo novas ferramentas para ajudar no esforço do estudo da eletrodinâmica da ionosfera e seus mecanismos de geração do spread $\mathrm{F}$.

O desenvolvimento de técnicas para fazer a detecção de irregularidades de plasma na ionosfera irão trazer benefícios à comunidade científica, por ser mais uma ferramenta para estudar a ionosfera. O estudo estatístico e observacional, que servirá de suporte ao aprimoramento do modelo empírico de spread $\mathrm{F}$ tem sua importância no esforço da comunidade científica de conhecer os mecanismos de geração de irregularidades do plasma ionosférico equatorial e de baixas latitudes para implementação de programas de previsão do Clima Espacial e de tempo real, assim como modelos de alerta de cintilação tão necessários hoje em dia para as telecomunicações e a navegação, assim evitando ou aminorando o desastre/catástrofe gerado por Efeitos Espaciais.

\section{Discussões e Conclusões}

A busca por novas técnicas para obter medidas mais precisas e eficientes e a criação de técnicas que permitam estudar melhor as irregularidades ionosféricas, obtendo consequentemente melhor compreensão de sua eletrodinâmica, podendo obter assim por análise dos dados até os mecanismos reais, ainda não puramente 
Desenvolvimento de uma técnica de detecção de irregularidades ionosféricas.

compreendidas, da geração das irregularidades, motivam fortemente a realização deste trabalho.

O estudo das irregularidades ionosféricas e seu real entendimento é de grande relevância, visto que, a ocorrência desse fenômeno causa interferência em sinais de rádio, impactando na área de telecomunicação e na navegação. Logo, o desenvolvimento de técnicas capazes de detectar essas estruturas irregulares aliado ao poder de conhecê-las melhor, é condição primordial para melhor transmissão de sinais na telecomunicação, na navegação, etc.

Este trabalho visa buscar compreender os mecanismos geradores de spread $F$ nas latitudes equatoriais e em baixas latitudes, fazendo uso de multi-instrumentos para o desenvolvimento de uma técnica que detecte esses mecanismos quando presentes. Um mecanismo de geração de perturbação é a exigência para a iniciação da instabilidade Rayleigh-Taylor Gravitacional (GRT) para desenvolver ESF (Haerendel et al., 1992). Procurando por mecanismos geradores de spread $\mathrm{F}$ muitos autores têm sugerido que as ondas de gravidade atmosféricas (AGW) geradas em altitudes troposféricas e se propagando para altitudes ionosféricas seriam o ponto de partida para gerálos (Kelley et al., 1981; Hysell et al., 1990; Kherani et al 2009; Abdu et al., 2009a; Takahashi et al., 2010).

Takahashi et al. (2010) observou uma estrutura senoidal na emissão de depleção airglow Ol 630 nm e oscilações verticais na altura da base da camada $\mathrm{F}$ em associação com estruturas de irregularidades bottom-type que poderiam indicar a AGW como o mecanismo de geração de bolhas de plasma. Keskinen et al. (2009) concluíram que a ondas de gravidade com propagação zonal, na presença de ventos zonais de maré, são necessários para a excitação de bolhas na ionosfera equatorial.

Este trabalho possibilitará além de uma técnica para detecção de bolhas, poderá apresentar uma contribuição para identificar os principais fatores e agentes responsáveis no início e geração das bolhas de plasma equatoriais.

\section{Referências}

Abdu, M.A., E.A. Kherani, I.S. Batista and J.H.A. Sobral (2009a), Equatorial evening prereversal vertical drift and spread F suppression by disturbance penetration electric fields, Geophys. Res. Lett., 36, L19103, doi:10.1029/2009GL039919.

Abdu, M.A., E.A. Kherani, I.S. Batista, E.R. de Paula, D.C. Fritts and J.H.A. Sobral (2009b), Gravity Wave initiation of equatorial spread F/plasma bubble irregularities based on observational data from SpreadFEx campaign, Ann. Geophys., 27, 2607-2622.

Fejer, B.G., L. Scherliess and E.R. de Paula (1999), Effects of the vertical plasma drift velocity on the generation and evolution of equatorial spread F, J. Geophys. Res., 104(A9), Pages 19,859-19,869.

Haerendel G., J.V. Eccles and S. Çakir (1992), Theory for modeling the Equatorial Evening lonosphere and the Origin of the Shear in the Horizontal Plasma Flow, J. Geophys. Res., 97(A2), Pages 1209-1223, doi:10.1029/91JA02226.

Hysell et al., (1990), Seeding and layering of equatorial spread F by gravity waves, J. Geophys. Res.,95, 1725317260.

Kelley M.C., M. F. Larsen, and C. LaHoz (1981), Gravity Wave initiation of equatorial spread F: A case study, J. Geophys. Res., 86, 9087-9100, doi:10.1029/JA086iA11p9087.

Kherani E.A. et al (2009), The impact of gravity waves rising from convection in the lower atmosphere on the generation and nonlinear evolution of equatorial bubble, Ann. Geophys., 27, 1657-1668. 
Silva, E. N.; Cueva, R. Y. C.

Keskinen et al. (2009), Three-dimensional nonlinear evolution of equatorial ionospheric bubbles with gravity waves seeding and tidal wind effects, Geophys. Res. Lett., 36, L12102, doi:10.1029/2009GL037892.

Narayanan, V. Lakshmi, et al. (2012), On the importance of wave-like structures in the occurrence of equatorial plasma bubbles: A case study, J. Geophys. Res., 117, A01306, doi:10.1029/2011JA017054.

Takahashi et al. (2010), Equatorial ionosphere bottom-type spread $\mathrm{F}$ observed by $\mathrm{Ol} 630.0 \mathrm{~nm}$ airglow imaging, Geophys. Res. Lett., 37, L03102, doi:10.1029/2009GL041802.

Thampi, S.V. et al. (2009), First observations of large-scale wave structure and equatorial spread $F$ using CERTO radio beacon on the C/NOFS satellite, Geophys. Res. Lett., 36, L18111, doi:10.1029/2009GL039887.

Tsunoda R.T. (2008), Satellite traces: An ionogram signature for large-scale wave structure and a precursor for equatorial spread F, Geophys. Res. Lett., 35, L20110, doi:10.1029/2008GL035706.

Tsunoda R.T. (2010a), On seeding equatorial spread F: Circular gravity waves, Geophys. Res. Lett., 37, L10104, doi:10.1029/2010GL043422.

Tsunoda R.T. (2010b), On seeding equatorial spread F during solstices, Geophys. Res. Lett., 37, L05102, doi:10.1029/2010GL042576.

Tsunoda, R. T., D. M. Bubenik, S. V. Thampi, and M. Yamamoto (2010), On large-scale wave structure and equatorial spread $F$ without a post-sunset rise of the $F$ layer, Geophys. Res. Lett., 37, L07105, doi:10.1029/2009GL042357.

Tsunoda R.T., et al. (2011), On seeding, large-scale wave structure, equatorial spread $F$, and scintillation over Vietnam, Geophys. Res. Lett., 38, L20102, doi:10.1029/2011GL049173.
Valladares, C. E., J. Villalobos, R. Sheehan, and M. P. Hagan (2004), Latitudinal extension of low-latitude scintillations measured with a network of GPS receivers, Ann. Geophys., 22, 3155 -3175, doi:10.5194/ angeo-223155-2004.

Weber, E. J., Basu, S., Bullett, T. W., et al.: Equatorial plasma depletion precursor signatures and onset observed at 11 south of the magnetic equator, J. Geophys. Res., 101, 26829, 1996.

Woodman, R. F., and C. LaHoz (1976), Radar observations of $\mathrm{F}$ region equatorial irregularities, J. Geophys. Res., 81, 5447-5466. 